\title{
A stochastic optimal control solution to the energy management of a microgrid with storage and renewables
}

\author{
Andrea Belloni, Luigi Piroddi, Maria Prandini
}

\begin{abstract}
The presence of renewable energy generators in a microgrid calls for the usage of storage units so as to smooth the variability in energy production. This work addresses the optimal management of a battery in a microgrid including a wind turbine facility. A Markov chain model is employed to predict the wind power production and the optimal management of the energy storage element is formulated as a stochastic optimal control problem. An approximate dynamic programming approach resting on system abstraction is then proposed for control policy design. Some numerical examples show the effectiveness of the approach.
\end{abstract}

\section{INTRODUCTION}

The classical paradigm of centralized energy generation, with large power plants serving vast geographic areas, is currently challenged by the widespread exploitation of distributed and stochastic energy sources, such as renewables. Different solution concepts are required to deal with this new scenario. The great variability and limited predictability in the energy production from renewable energy sources may easily cause demand-production unbalance and calls for the adoption of energy storage elements jointly with appropriate energy management strategies to mitigate the effect of such fluctuations.

In the present work we address the energy management of a small microgrid (see, e.g, [1], [2], [3], [4]), that includes a wind turbine, a load and an energy storage element (a battery). The microgrid is connected to the distribution grid, which accommodates for unbalances between the supplied and consumed power.

The wind energy is primarily employed to supply the load, and is possibly supplemented by the energy provided by the battery and/or the main grid. Energy in excess is either sold to the grid or stored in the battery. The energy cost is time dependent and the microgrid energy management problem consists in minimizing the cost of the energy exchanged with the main grid during some reference time horizon. The difficulty in predicting the power generated by the wind energy source typically forces the energy management operator to adopt conservative policies, such as storing energy for buffering purposes. The load is also subject to fluctuations, although of lesser importance.

The following assumptions are made throughout the paper:

- The microgrid energy management operator is concerned with the optimal energy management (cost min-

This work is partially supported by the European Commission under the project UnCoVerCPS, grant number 643921.

A. Belloni, L. Piroddi, M. Prandini are with the Dipartimento di Elettronica, Informazione e Bioingegneria of the Politecnico di Milano - Milano, Italy \{luigi.piroddi, maria.prandini\}@polimi.it; belloni.andegmail.com imization) of the system only. Grid stability and frequency control are under the control of the main grid.

- The flow balancing of the reactive power in the grid is ensured by the voltage stability control system. In view of this, only the active power is here considered.

- The microgrid energy management operator has full information on the system, so that a fully centralized control system can be designed.

The main control knob in this framework is provided by the battery unit. An energy storage element can alleviate congestion problems on the energy transmission and distribution lines, as occur, e.g., when a peak in wind power production corresponds to a moderate demand. It can also serve to smooth out the irregular energy production [5], [6], and level the load peaks by (partially) shedding the energy usage in the microgrid through time-shifting. This is particularly relevant in the presence of feed-in-tariffs, which tend to favor the consumption of auto-produced energy. Several attempts to manage the complex interplay between the highly variable wind power generation and the storage element are documented in the literature, mainly based on Model Predictive Control (MPC) [7], [8], [9], or stochastic programming techniques [10].

Here, the main objective of the control design is to optimally use the battery, so as to complement the wind turbine in fulfilling the load requirement, reducing overall the energy cost towards the main grid along a medium term horizon of some days. More specifically, the battery should be managed so as to appropriately modulate in time the microgrid energy exchanges with the main grid depending on the time-varying charged price of the energy. To this purpose, we adopt a stochastic optimal control approach integrating a Markov chain model for wind power prediction as suggested in [11]. The microgrid is described as a discrete time stochastic system with both discrete and continuous state variables and the battery charge/discharge as control input. A finite-horizon average cost function is minimized subject to constraints on the state and rate of charge of the battery unit. In principle, a Dynamic Programming (DP) solution can be derived to determine an optimal state feedback policy. In practice, some approximation is required to solve the DP equations. Interestingly, the only approximation that one needs to introduce consists in the abstraction of the stochastic hybrid system to a purely discrete one via quantization of the control input. As the quantization becomes finer, one recovers the optimal solution. Moreover, the approximation is exact if one constrains the control input to take only the chosen quantized values. This is not generally the case when 
adopting approximate DP solutions, [4].

\section{MICROGRID DESCRIPTION AND MODELING}

The system under consideration is schematically represented in Figure 1. It consists of an electrical load served by a wind turbine complementing the main grid, and a battery that can be used to accommodate for the variability of the wind-generated power as well as the load itself.

The power balance in the system is given by:

$$
P_{g}(t)=P_{w}(t)+P_{l}(t)+P_{b}(t),
$$

where $P_{g}$ is the net power exchanged with the main grid (positive when entering the microgrid), $P_{w} \leq 0$ denotes the power produced by the wind turbine, $P_{l} \geq 0$ is the power absorbed by the load, and $P_{b}$ is the power exchanged with the battery (positive when charging and negative otherwise). Note that, in our framework, the only control input is $P_{b}$, whereas both $P_{w}$ and $P_{l}$ can be viewed as disturbances since we consider only uncontrollable loads. In the sequel we shall derive a model for each component in terms of energy per time slot (of duration $T$ ).

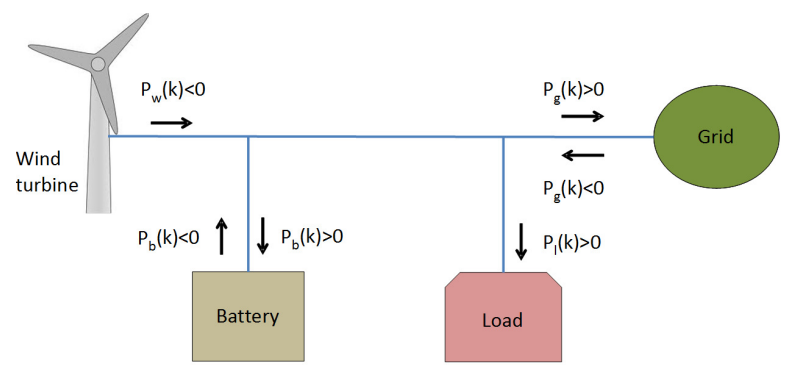

Fig. 1. System configuration.

\section{A. Wind energy prediction model}

A wind turbine is used to convert the kinetic energy of an air mass in motion to electric energy. Energy is actually produced if the wind speed exceeds a cut-in value. To avoid machine damage, the rotor must be stopped if the wind speed exceeds a cut-off value. Between these two extremal points, the produced power $P_{w}$ depends on the wind speed $v_{w}$ according to a characteristic curve of the type depicted in Figure 2. In Figure 2, $v_{n}$ denotes the nominal turbine speed,

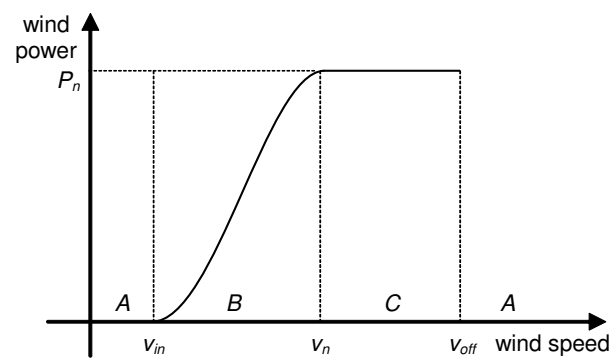

Fig. 2. Characteristic power curve for a wind turbine.

over which the pitch control system operates to preserve the maximum nominal power, while $v_{i n}$ and $v_{\text {off }}$ are the cutin and cut-off speeds, respectively. The wind power can be calculated as follows:

$$
P_{w}= \begin{cases}0, & v<v_{i n} \text { or } v>v_{o f f} \\ P_{m}(v), & v_{i n} \leq v<v_{n} \\ P_{n}, & v_{n} \leq v<v_{o f f}\end{cases}
$$

where $P_{m}(v)$ denotes the maximum power achievable for wind speed values lower than $v_{n}$, while $P_{n}$ is the nominal power. $P_{m}(v)$ is a continuous function of $v$ that can be accurately approximated e.g. via a polynomial expansion.

Both physical and statistical models have been considered for wind power prediction in the literature, [12], [13], [14]. We are here mainly concerned with methods of the latter class, which aim at reproducing the statistical properties of the phenomenon extracting all the relevant information from available data. Classical time series analysis using autoregressive (AR) or autoregressive moving average (ARMA) models is appealing due to the reduced number of parameters and the simplicity of the parameter estimation, but the reported results are not particularly accurate [11]. A viable alternative is to model the phenomenon using a (stationary) discrete time Markov Chain (MC), where wind energy is discretized in a finite number of values $s_{1}, s_{2}, . ., s_{M}$, and the transition probabilities $p_{i j}$ from state $s_{i}$ to $s_{j}, i, j=$ $1, \ldots, M$, are defined.

To this purpose, the wind energy per time slot $T$ is first computed from wind speed data using (2). Then, it is uniformly quantized in the range $\left[\underline{E}_{w}, \bar{E}_{w}\right]$ with $M$ bins, each accounting for an interval of width $\Delta E_{w}=\frac{\bar{E}_{w}-\underline{E}_{w}}{M}$, $\underline{E}_{w}$ and $\bar{E}_{w}$ being the minimum and maximum energy generated by the wind turbine in a time slot of duration $T$. The system is in $s_{i}$ if the current energy is in the range $\left[\underline{E}_{w}+(i-1) \Delta E_{w}, \underline{E}_{w}+i \Delta E_{w}\right]$. Finally the transition probabilities $p_{i j}$ can be estimated as follows:

$$
\hat{p}_{i j}=\frac{n_{i j}}{\sum_{k} n_{i k}}, \quad i, j=1, \ldots, M,
$$

where $n_{i j}$ is the number of state transitions from $s_{i}$ to $s_{j}$ in the state sequence. An example of transition probability matrix obtained on actual wind data is represented in Figure 3. The system has a strong tendency to remain in the current state (see the high probability values on the main diagonal of the matrix). This is particularly true for the extremal states, corresponding to $0\left(s_{1}\right)$ or maximal energy $\left(s_{M}\right)$.

\section{B. The battery}

In the energy management of the microgrid on a medium term horizon, the battery is essentially employed as a means to shift the load requirements over time. Electrical batteries are characterized by the power and energy they can supply. Other important features are efficiency, lifetime, operation temperature, charge and discharge characteristics, self-discharge. The State Of Charge (SOC) is a dimensionless parameter that expresses the amount of energy stored in the battery in percentage with respect to the maximum storable charge: $S O C(t)=100 \int_{t_{0}}^{t} I(t) d t / \int_{t_{0}}^{\infty} I(t) d t$, where $I(t)$ is the charging current and the battery is assumed completely 


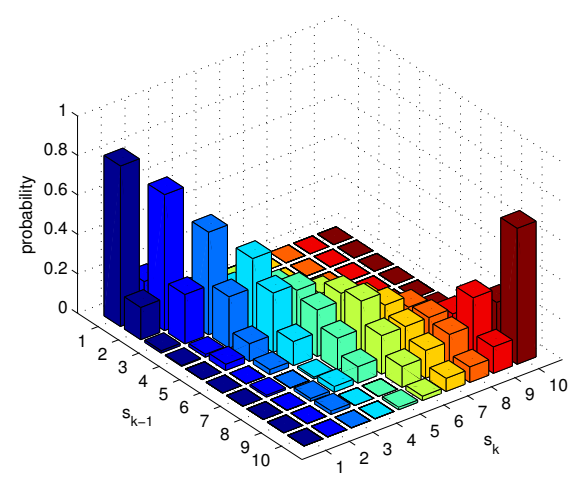

Fig. 3. Pictorial representation of the transition probability matrix.

discharged at $t_{0}$. Estimating precisely the SOC is obviously crucial for the optimal management of the battery (see, e.g., [15], [16]). The energy $E_{b}$ stored in the battery, together with the charge power $P_{b}$, provide an equivalent information to the variables $S O C$ and $I$. The stored energy is defined as $E_{b}(t)=\int_{t_{0}}^{t} P_{b}(t) d t$, from which the following discretized equation can be derived:

$$
E_{b}(k+1)=E_{b}(k)+P_{b}(k) T,
$$

where $k$ is the discrete time index, $P_{b}(k)$ denotes the charge power which is kept constant over the time slot $[k T,(k+$ 1)T], while $E_{b}(k)$ is the actual value of the energy stored at time $k T$. The battery is subject to the following physical constraints, $\underline{P}_{b} \leq P_{b}(k) \leq \bar{P}_{b}, \underline{E}_{b} \leq E_{b}(k) \leq \bar{E}_{b}$, where $\underline{P}_{b}$ and $\bar{P}_{b}$ are the maximum discharge and charge values, and $\underline{E}_{b}$ and $\bar{E}_{b}$ are bounds on the battery capacity. Typically, the minimum battery capacity is set strictly larger than zero $\left(\underline{E}_{b}>0\right)$ for battery management purposes, since complete battery discharges degrade its behavior [7]. Similarly, the maximum capacity $\bar{E}_{b}$ is limited to $90 \%-95 \%$ of the actual maximum energy that could physically be stored in the battery.

\section{Load model}

The prediction of the load request in terms of electrical energy is crucial for performing an adequate planning. Short term predictions are usually obtained by processing load time histories as well as meteorological data (e.g., temperature and humidity), and must take into consideration the class of users (e.g., residential, commercial or industrial). Both additive [17], [18], and multiplicative [19] models are documented in the literature to combine the different load components (e.g., the nominal load associated with the considered user class, the additional load that depends on the atmospheric conditions, a term accounting for the effect of energy price, noise). A simpler approach is adopted here, considering a nominal load profile with an additive noise: $E_{l}(k)=$ $\bar{E}_{l}(k)+\epsilon(k)$, where $\bar{E}_{l}(k)$ represents the nominal value of the requested energy per time slot and $\epsilon(k)$ is a zero mean white Gaussian noise with variance $\sigma^{2}: \epsilon(k) \sim \mathcal{W} \mathcal{G} \mathcal{N}\left(0, \sigma^{2}\right)$.

\section{Electricity pricing}

The considered microgrid can purchase and sell electricity to the grid, the decisions regarding which practice to adopt being influenced by the price of electricity. The latter is generally variable during the day to influence the users' policy regarding electricity consumption with the general purpose of evening out the consumption peaks yielding a more uniform load profile. Furthermore, to disincentivize large energy requests in brief periods of time, the electricity price is increased when a given limit $\left(\bar{E}_{g}\right)$ is exceeded, introducing a penalty factor $g$.

Denoting with $p_{p}(k)$ the electricity purchase price at the $k$ th time slot and with $p_{s}$ the (constant) energy sale price, the total expense (or profit, if negative) at the $k$ th time slot is defined by the following function:

$$
C_{k}\left(E_{g}\right)=\left\{\begin{array}{l}
p_{s} E_{g}, E_{g}<0 \\
p_{p}(k) E_{g}, 0 \leq E_{g} \leq \bar{E}_{g} \\
p_{p}(k)\left[\bar{E}_{g}+g\left(E_{g}-\bar{E}_{g}\right)\right], E_{g}>\bar{E}_{g}
\end{array}\right.
$$

where $E_{g}$ is the energy exchanged with the grid in the $k$ th time slot. The energy sale price $p_{s}$ is lower than the purchase price $p_{p}(k)$ to incentivize the direct usage of auto-produced energy. As a side effect of the condition $p_{s}<p_{p}(k), C_{k}\left(E_{g}\right)$ is a convex function of the control input. This is easily seen given that $E_{g}(k)$ can be computed by integrating (1) over the $k$-th time slot and it is hence affine as a function of the charge/discharge battery rate $P_{g}(k)$ :

$$
E_{g}(k)=E_{w}(k)+E_{l}(k)+P_{b}(k) T .
$$

\section{THE OPTIMAL ENERGY MANAGEMENT PROBLEM}

The microgrid energy management task has a two-fold objective: guaranteeing full load satisfaction while minimizing the overall expense over some look-ahead time horizon $\left[t_{0}, t_{f}\right]$. The primary objective is achieved assuming that the grid can exactly cover any residual unbalance left by the wind turbine and battery system, as of equation (1). As for the second objective, the time horizon is divided into $N$ time slots of duration $T$ and the control input $P_{g}$, is set so as to minimize the following finite horizon average cost:

$$
\sum_{k=0}^{N-1} \mathbb{E}\left[C_{k}\left(E_{g}(k)\right)\right]
$$

where the energy exchanged with the grid in the $k$ th time slot $E_{g}(k)$ is given by (5), and the battery power exchange $P_{g}(k)$, $k=0,1, \ldots, N-1$, is subject to the following constraints on its instantaneous value and on its integral by way of $E_{b}(k)$ (see (3)): $\underline{P}_{b} \leq P_{b}(k) \leq \bar{P}_{b}, \underline{E}_{b} \leq E_{b}(k) \leq \bar{E}_{b}$, $k=1, \ldots, N-1$, and $E_{b}(N)=E_{b, \text { med }}$. The last equality constraint sets the final state of charge of the battery equal to $E_{b, \text { med }}$ which is half the maximal capacity, [5], [7], so as to guarantee maximum flexibility to the optimization algorithm at the beginning of the next control time horizon.

The cost function depends on the energy $E_{g}$ exchanged with the grid, which in turn depends on $E_{w}, E_{l}$, and $P_{b}$ (see (5)). The wind turbine is assumed to be controlled so as to yield the maximum power compatible with the wind conditions, and $E_{w}$ and $E_{l}$ are modeled as independent disturbances, for simplicity. The expectation in (6) is taken with respect to both these stochastic disturbances realizations. By optimally 
scheduling the charging and discharging of the battery according to the minimization of the cost (6), one determines when to buy and sell energy to the grid.

\section{DP-BASED SOLUTION}

The optimal energy management problem formulated in Section III is a finite horizon constrained optimization problem where some variables are subject to uncertainty. An heuristic solution approach to problems of this class has been proposed e.g. in [20]. Other solutions are based on a linear programming (LP) reformulation of the problem, such as [2], where a combination of LP with MPC is employed, or [21], which solves first the optimization problem assuming given load and wind power profiles, and then uses a second optimization layer on-line to tackle variations from the given profiles. An alternative line of approach to the problem is provided by Dynamic Programming (DP), [22], which is also pursued here. DP is a multi-stage resolution technique for sequential optimal control problems that can also account for the presence of stochastic disturbances. Examples of application of DP-based techniques to the energy optimization of a microgrid are given in [21], [4].

In view of a DP-based solution, the considered optimization problem is reformulated as follows [22]:

$$
\min _{\pi \in \Pi} J_{\pi}(x(0))
$$

subject to:

$$
\begin{aligned}
& x(k+1)=f_{k}(x(k), u(k), w(k)), k=0, \ldots, N-1 \\
& u(k)=\mu_{k}(x(k)) \in U_{k}(x(k)), k=0, \ldots, N-1 \\
& x(k) \in X, k=0, \ldots, N-1
\end{aligned}
$$

where $x(k) \in X$ is the system state, $u(k) \in U_{k}(x(k))$ is the control input (notice that $U_{k}(x(k))$ may in general be a function of the current state), and $w(k)$ is a disturbance term acting on the system, characterized by a probability distribution $\mathbb{P}_{k}(\cdot \mid x(k), u(k))$ which may depend on the current state and action.

The control policy $\pi$ is a sequence of state-action maps $\left\{\mu_{0}, \ldots, \mu_{N-1}\right\}$, and the control input at time $k$ is chosen according to $u(k)=\mu_{k}(x(k))$. A policy is admissible if $\mu_{k}(x) \in U_{k}(x)$ for all $x \in X$. The set of admissible policies is denoted $\Pi$. The cost function accumulates over time additively:

$$
J_{\pi}(x(0))=\mathbb{E}_{w}\left[g_{N}(x(N))+\sum_{k=0}^{N-1} g_{k}(x(k), u(k), w(k))\right],
$$

where $\mathbb{E}_{w}$ denotes the expectation with respect to the disturbance sequence $w(0), \ldots, w(N-1)$. Each time step provides an individual contribution to the overall cost, which depends on the current state and control action (as well as the disturbance), except the terminal cost $g_{N}$, which only depends on the final state. The cost function $J_{\pi}(x(0))$ depends on the initial state $x(0)$ and on the chosen control policy. In our case, we have:

$$
\begin{aligned}
& x_{1}(k+1)=x_{1}(k)+u(k) T \\
& x_{2}(k+1)=w_{1}(k)
\end{aligned}
$$

where $x_{1}(k)$ is the battery charge, $x_{2}(k)$ is the energy generated by the wind turbine, $u(k)$ is the battery charging/discharging power, and $w_{1}(k)$ is a disturbance term modeled by the probability distribution $\mathbb{P}\left(w_{1}(k)=s_{j} \mid x_{2}(k)=\right.$ $\left.s_{i}\right)=p_{i j}$, as resulting from the MC model of the wind energy. Notice that the state $x=\left(x_{1}, x_{2}\right)$ is hybrid, being $x_{1}$ continuous and $x_{2}$ discrete. The state space is defined as $X=X_{1} \times X_{2}$, where $X_{1}=\left\{x_{1} \in \mathbb{R} \mid: \underline{E}_{b} \leq x_{1} \leq \bar{E}_{b}\right\}$ and $X_{2}=\left\{s_{1}, s_{2}, . ., s_{M}\right\}$. Correspondingly, the control space is defined as follows:

$$
U_{k}(x)=\left\{u \in R \mid: \frac{\underline{E}_{b}-x_{1}}{T} \leq u \leq \frac{\bar{E}_{b}-x_{1}}{T}\right\} .
$$

The cost term $g_{k}$ is defined as follows:

$$
g_{k}=\left\{\begin{array}{l}
C_{k}\left(x_{2}(k)+w_{2}(k)+u(k) T\right), k=0, \ldots, N-1 \\
\alpha\left(x_{1}(k)-E_{b, \text { med }}\right)^{2}, k=N
\end{array}\right.
$$

where

$$
w_{2}(k)=\bar{w}_{2}(k)+\epsilon(k),
$$

$\bar{w}_{2}$ representing the nominal (daily) load profile and $\epsilon$ being a Gaussian white noise term. As stated previously, $w_{1}$ and $w_{2}$ are assumed to be independent. The terminal cost implements the requirement on the final state of charge of the battery as a soft constraint ( $\alpha>0$ should be set sufficiently large to enforce this condition).

The DP solution to the considered optimal multi-stage optimization problem can be obtained by applying the value iteration approach. This involves determining the value functions $V_{k}: X \rightarrow \mathbb{R}, k=0,1, \ldots, N$, as described below, and then solving a minimization problem at each stage $k$ so as to compute an optimal admissible policy $\pi^{\star}=\left\{\mu_{0}^{\star}, \ldots, \mu_{N-1}^{\star}\right\} \in \Pi$. More precisely, we have to set $V_{N}(x)=g_{N}(x), x \in X$, and compute the value functions $V_{k}: X \rightarrow \mathbb{R}, k=0,1, \ldots, N-1$ via the backward recursion: $V_{k}(x)=\min _{u \in U_{k}(x)} E_{w(k)}\left[g_{k}(x, u, w(k))+V_{k+1}\left(f_{k}(x, u, w(k))\right]\right.$. Then,

$$
\mu_{k}^{\star}(x) \in \arg \min _{u \in U_{k}(x)} V_{k}(x), k=0,1, \ldots, N-1 .
$$

Notice that the expectations involved in the value functions computations are taken with respect to a MC disturbance and a Gaussian noise. Hence, they admit an analytic expression for each given $x$ and $u$ pair of values. However, the state $x=$ $\left(x_{1}, x_{2}\right)$ has a continuous component $x_{1}$ and the control input $u$ is continuous as well, which hampers the exact solution to the DP equations. One has then to resort to an approximate solution. Interestingly, the structure of the problem simplifies this task. Note that $x_{1}$ is the battery charge and as such it is obtained by integrating the control input $u$ representing the rate of charge/discharge of the battery. It then suffices to quantize the input $u$ to get a discrete abstraction of the system and solve the DP equations numerically. The obtained solution is actually exact if the input $u$ is allowed to take values only in the chosen quantized control space. If the control input were to be freely selected, then, the obtained solution would only represent an approximation of the optimal control policy, whose accuracy improves as the quantization gets finer. 


\section{A NUMERICAL CASE STUDY}

We next detail some characteristics of a numerical case study introduced to assess the performance of the proposed microgrid energy optimization strategy.

Regarding the wind turbine description, the following parameters have been employed in (2): $P_{n}=300 \mathrm{~kW}, v_{i n}=$ $2 \mathrm{~m} / \mathrm{s}, v_{n}=14 \mathrm{~m} / \mathrm{s}, v_{\text {off }}=20 \mathrm{~m} / \mathrm{s}$. Furthermore, the maximum power curve in the $\mathrm{B}$ region of Figure 2 has been approximated as $\hat{P}_{m}(v)=a_{0}+a_{1} v+a_{2} v^{2}$, with $a_{0}=-119.22, a_{1}=-64.67$ and $a_{2}=2.53$.

A data series containing average wind velocity measurements over a period of 4 months on a 10 minute scale was used for deriving the transition probability matrix represented in Figure 3. A sub-sampling has been performed to obtain a series of hourly data, averaging the previous data over each hour period. The resulting 4320 points have been quantized in $M=10$ bins of amplitude $\Delta E_{w}=33 \mathrm{kWh}$. The corresponding transition probability matrix is represented in Figure 3.

As for the electricity purchase price, we consider the three tariffs for residential consumers adopted by the Italian electric utility, depending on the time of the day:

F1) $0.21 € / \mathrm{kWh}$ from 23 to 7

F2) $0.27 € / \mathrm{kWh}$ from 7 to 8 and from 19 to 23

F3) $0.30 € / \mathrm{kWh}$ from 8 to 19

Figure 4 displays the corresponding daily price profile. The (constant) energy sale price $p_{s}$ was set to $0.10 € / \mathrm{kWh}$.

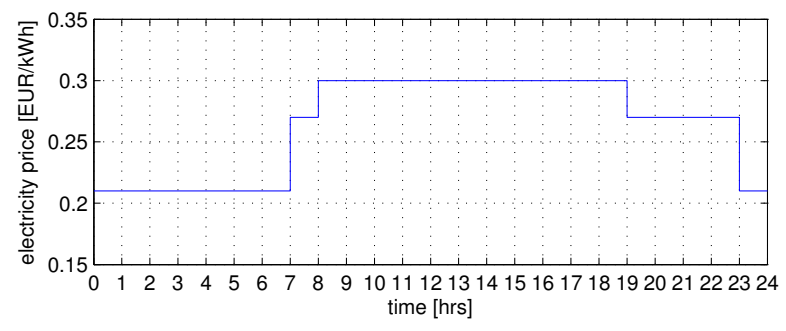

Fig. 4. Daily electricity price profile.

A group of lead batteries is considered, with a maximum physical capacity of $E_{\max }^{c}=1 \mathrm{MWh}$, for which the energy bounds are defined as $\underline{E}=0.2 \mathrm{MWh}$ and $\bar{E}=0.9 \mathrm{MWh}$, while the power limits are set to $\underline{P}_{b}=-0.2 \mathrm{MW}$ and $\bar{P}_{b}=$ $0.2 \mathrm{MW}$.

The considered nominal load profile (see Figure 5) pertains to a block of residential flats on a working day and is characterized by two peak requests, in the early morning and in the evening hours. A peak request of $0.233 \mathrm{MWh}$ occurs around 8 p.m. The fluctuation $\epsilon(k)$ around the nominal load profile in (7) is a white Gaussian noise $\mathcal{W G \mathcal { N }}\left(0, \sigma^{2}\right)$ with $\sigma=0.02 \mathrm{MWh}$.

\section{A. Validation of the optimal policy}

The optimal policy provides a state-action map at every hour of the considered time horizon. A graphic representation of the optimal policy at three different times of the day is depicted in Figure 6. As a general rule, given the load and energy price profiles, the optimal policy tends to charge the

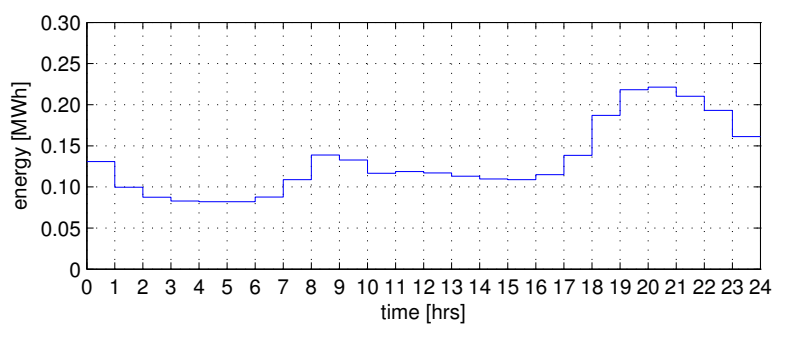

Fig. 5. Nominal daily load energy profile.
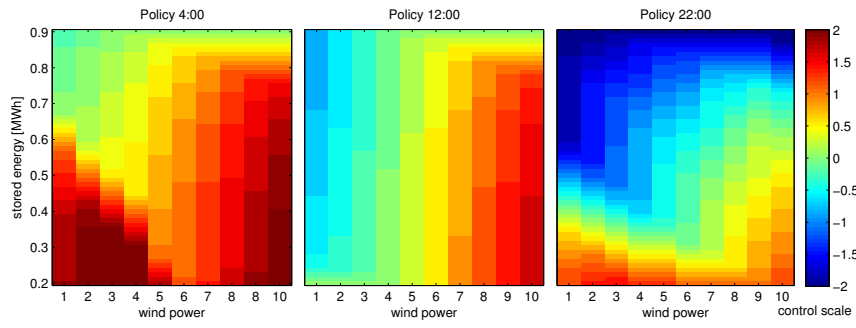

Fig. 6. Optimal state-action maps at different hours of the day.

battery in the early hours of the day (Figure 6, left), when the energy price and the load requirement are low, and uses the stored energy later in the day (Figure 6, middle), when the energy price and the load requirement are high. Wind energy is preferably stored than sold at the beginning of the day, whereas the reverse occurs during daylight hours (if any excess energy remains after serving the load). Finally, in the evening hours (Figure 6, right) the battery is recharged to meet the final state condition. More in detail, notice in Figure 6 (left) that for low wind power conditions the battery is charged progressively less as the wind increases. This can be explained by considering the inertia in the MC model of the wind power. Indeed, since persistence wind conditions are to be expected it becomes less urgent to charge the battery as the level of the wind increases. Large charging powers also occur for strong wind levels. Also, fewer energy is stored in the battery as the battery level increases. The large variability of the wind conditions during the day results in a large spread of the economic results of the policy. To see this, the optimal policy has been tested on $10^{4}$ realizations of the stochastic variable $w=\left[w_{1} w_{2}\right]^{T}$ over the entire time horizon. Figure 7 displays the resulting cost histograms for different initial wind conditions. Negative costs correspond to profits. Since the wind power MC has a certain inertia, initially low wind power values generally determine a large requirement of energy from the main grid during the day, rarely resulting in actual profits, and viceversa. Sampling $x_{2}(0)$ uniformly over $S$ and generating the load profile according to (7) yields a wide spectrum of solutions, ranging from a profit of nearly $400 €$ (strong wind conditions and minimal load request) to an overall cost of nearly $950 €$ (no wind conditions and maximal load request), with an average cost of $241 €$.

\section{B. Policy optimization in different conditions}

To evaluate the effectiveness and utility of the microgrid components, the optimization has been repeated in different 

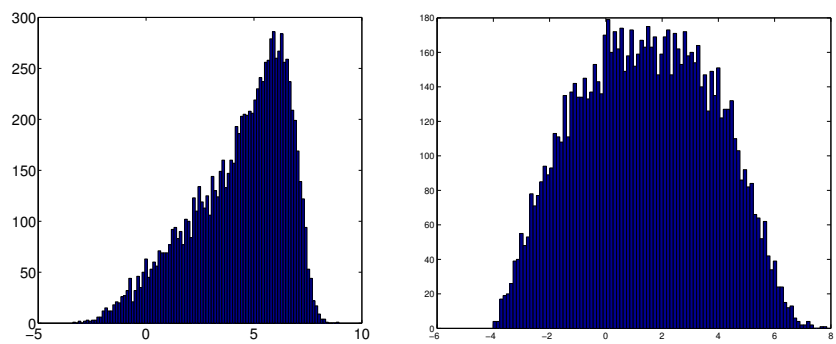

Fig. 7. Histograms of the total cost (in $10^{2} €$ ), for $10^{4}$ realizations, starting from $x_{1}(0)=E_{m e d}$ and $x_{2}(0)=s_{3}$ (left) or $x_{2}(0)=s_{8}$ (right).

conditions and the overall average cost calculated. For example, in the absence of both the storage element and the wind turbine, and assuming a nominal load requirement, a total cost of $846,6 €$ is estimated. The wind turbine yields some saving (an average profit of $239 €$ is obtained for constant strong wind conditions, whereas a cost of $594 €$ results if the wind falls completely after 10 a.m.), although the wind power is not exploited in full (power in excess cannot be stored and must be sold to the grid at the current price). In the absence of wind generation, the use of the battery alone (pre-loaded at $E_{m e d}$ ) guarantees a $7 \%$ savings over the basic reference case ( $795 €$ of energy cost), by allowing the user to buy energy at the most convenient time. If both the storage element and the wind turbine are present the battery is of limited use for constant strong wind conditions, since it saturates early on, yielding an average profit of 254 $€$. Conversely, in conditions of (strong) wind only up to 10 o'clock the battery has a more decisive role guaranteeing $100 €$ of savings compared to the corresponding condition without storage (average cost of $494 €$ ).

Figure 8 shows the various power profiles $\left(E_{g} / T, P_{b}, E_{l} / T\right.$, $\left.E_{w} / T\right)$, as well as the storage capacity usage $\left(E_{b}\right)$, over a period of three days, with varying wind conditions.

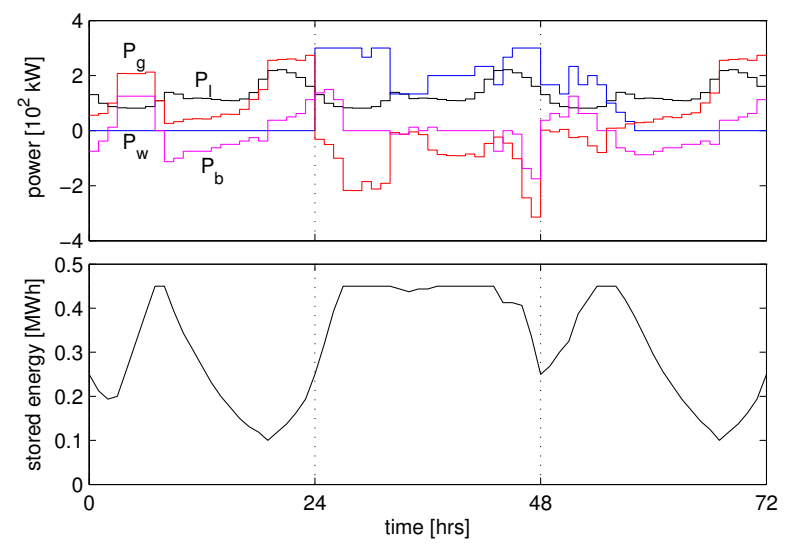

Fig. 8. Power profiles (top) and storage level (bottom) for a 3-day realization with varying wind conditions (no wind on the $1^{\text {st }}$ day, constant strong wind on the $2^{\text {nd }}$ day, partially windy on the $3^{\text {rd }}$ day).

The maximum cost incurred by the plant is greatly influenced by bounds on the maximum amount of energy per hour that can be bought from the grid $\left(\bar{E}_{g}\right)$. Indeed, in the absence of such bounds the worst case cost amounts to $845 €$. This figure grows to $948 €$ if $\bar{E}_{g}=300 \mathrm{kWh}$, and to as much as $1362 €$ if such limit is further halved.

\section{REFERENCES}

[1] P. Stluka, D. Godbole, and T. Samad, "Energy management for buildings and microgrids," in Conf. on Decision and Control \& European Control Conf., Orlando, USA, Dec. 2011, pp. 5150-5157.

[2] A. Parisio and L. Glielmo, "Energy efficient microgrid management using model predictive control," in Conf. on Decision and Control \& European Control Conf., Orlando, USA, Dec. 2011, pp. 5449-5454.

[3] R. Minciardi and R. Sacile, "Optimal control in a cooperative network of smart power grids," IEEE Systems Journal, vol. 6, no. 1, pp. 126133, 2011.

[4] F. Borghesan, R. Vignali, L. Piroddi, M. Prandini, and M. Strelec, "Approximate dynamic programming-based control of a building cooling system with thermal storage," in $4^{\text {th }}$ European Innovative Smart Grid Technologies Conference, Copenhagen, Denmark, October 6-9 2013.

[5] S. Teleke, M. E. Baran, A. Q. Huang, S. Bhattacharya, and L. Anderson, "Control strategies for battery energy storage for wind farm dispatching," IEEE Trans. on Energy Conversion, vol. 24, no. 3, pp. 725-732, Sept. 2009.

[6] T.-L. Pan, H.-S. Wan, and Z.-C. Ji, "Stand-alone wind power system with battery/supercapacitor hybrid energy storage," Int. J. of Sustainable Engineering, vol. 7, no. 2, pp. 103-110, 2014.

[7] S. Teleke, M. E. Baran, S. Bhattacharya, and A. Q. Huang, "Optimal control of battery energy storage for wind farm dispatching," IEEE Trans. on Energy Conversion, vol. 25, no. 3, pp. 787-794, Sept. 2010.

[8] L. Xie, Y. Gu, A. Eskandari, and M. Ehsani, "Fast MPC-based coordination of wind power and battery energy storage systems," $J$. Energy Eng., vol. 138, no. 2, pp. 43-53, 2012.

[9] A. Khatamianfar, M. Khalid, A. Savkin, and V. Agelidis, "Wind power dispatch control with battery energy storage using model predictive control," in IEEE Int. Conf. on Control Applications (CCA), Dubrovnik (Croatia), 3-5 Oct. 2012, pp. 733-738.

[10] Y. Yuan, Q. Li, and W. Wang, "Optimal operation strategy of energy storage unit in wind power integration based on stochastic programming," IET Renewable Power Generation, vol. 5, no. 2, pp. 194-201.

[11] G. Papaefthymiou and B. Klockl, "MCMC for wind power simulation," IEEE Trans. on Energy Conversion, vol. 23, no. 1, pp. 234-240.

[12] Y.-K. Wu and J.-S. Hong, "A literature review of wind forecasting technology in the world," in IEEE Power Tech, Lausanne, July 2007 , pp. 504-509.

[13] J. Taylor, P. McSharry, and R. Buizza, "Wind power density forecasting using ensemble predictions and time series models," IEEE Trans. on Energy Conversion, vol. 24, no. 3, pp. 775-782, Sept. 2009.

[14] U. Firat, S. Engin, M. Saraclar, and A. Ertuzun, "Wind speed forecasting based on second order blind identification and autoregressive model," in $9^{\text {th }}$ Int. Conf. on Machine Learning and Applications (ICMLA), Dec. 2010, pp. 686-691.

[15] B. Xiao, Y. Shi, and L. He, "A universal state-of-charge algorithm for batteries," in $47^{\text {th }}$ ACM/IEEE Design Automation Conference (DAC), June 2010, pp. 687-692.

[16] Z. Wang, J. Xu, and T. Wang, "The online monitoring system software design and the SOC estimation algorithm research for power battery," in IEEE Int. Conf. on Vehicular Electronics and Safety (ICVES), July 2013, pp. 89-92.

[17] E. A. Feinberg and D. Genethliou, "Load forecasting," in Applied Mathematics for Restructured Electric Power Systems, ser. Power Electronics and Power Systems, J. H. Chow, F. F. Wu, and J. Momoh, Eds. Springer US, 2005, pp. 269-285.

[18] H. Chen, C. Canizares, and A. Singh, "ANN-based short-term load forecasting in electricity markets," in IEEE Power Engineering Society Winter Meeting, vol. 2, 2001, pp. 411-415.

[19] S. Rahman, "Formulation and analysis of a rule-based short-term load forecasting algorithm," Proc. of the IEEE, vol. 78, no. 5, pp. 805-816.

[20] C. Wang and M. Nehrir, "Power management of a stand-alone wind/photovoltaic/fuel cell energy system," IEEE Trans. on Energy Conversion, vol. 23, no. 3, pp. 957-967, Sept. 2008.

[21] T. H. Pham, F. Wurtz, and S. Bacha, "Optimal operation of a PV based multi-source system and energy management for household application," in IEEE Int. Conf. on Industrial Technology (ICIT), Feb. 2009, pp. 1-5.

[22] D. P. Bertsekas, Dynamic Programming and Optimal Control. Massachusetts Institute of Technology, 2005. 